\section{Predicting protein function and annotating complex pathways with machine learning}

Dr Daisuke Kihara's team at
Purdue University have created
novel computational approaches
for predicting protein functions.
Instead of following a one-
protein-one-function approach,
their algorithms can predict the
functional relationships of entire
groups of proteins related to a
specific biological process. The
team has also expanded into
mining oversighted or previously
unknown proteins that have
multiple, independent functions.
The team's methods challenge
the logic behind conventional
protein function studies and
propose tools that may better
capture the complicated
nature of protein interactions
in biological processes.

\section{ROBLEMS WITH PREVIOUS}

roteins are the main working Units orbiology. Identifying and understanding what proteins do is crucial for biologists hoping to solve
the complex interactions and systems that the complex interactions and systems that dive celluar processes. Alh hygh proten function needs to be ultimately valid ted first need a hypothesis in order to design assays, which can then define the probable function of a prote

BIOINFORMATICS FOR

PREDICTING PROTEIN FUNCTION

Biologists can build such hypotheses

of gene function with computers.

As genome sequencing becomes

routine in experimental laboratories,

computational gene function prediction

has also become increasingly importan

Computational methods are very

suitable for function prediction because function information of a gene can be inferifom a database search that identifies simlanty between the gene and Sequence similarity Local Alignment Searh Tol (BAST) is one such method that searches agains all previously recorded sequences and suggests a scored list of possib roles for it.
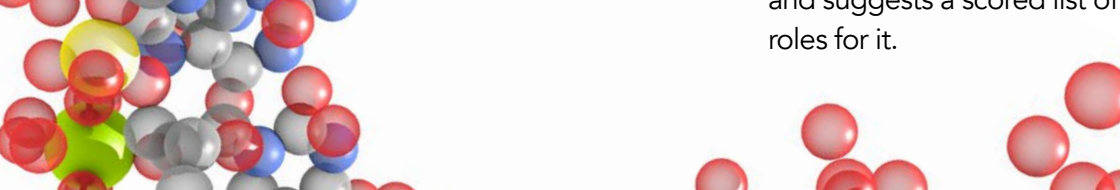

\section{COMPUTATIONAL METHODS}

always, existing bioinformatic tools can't and often end up incin function accurately proteins within a biological system. Protein function prediction high sequence similarity is detected, bu heir accuracy falls quickly for sequences with lower similarities. For example, similarity scores fall below a certain level. Moreover, in many cases traditional methods do not annotate any function if highly similar sequences are not found, leaving many genes unannotated. In addition, other metrics such as similarity in three-dimensional structure, gene expression, or interaction data could be used. However, each of these metrics are often missing for many proteins under investigation, and so have limited reliable research.

\section{NEW TOOLS FOR}

Recently, several new protein annotation methods have been developed to such developer is Dr Daisuke Kihara from Purdue University, who develops function prediction methods with new logical frameworks. In 2009, his team created an automated predictive algorithm, (ESG) method, which runs a continual (E) search. From each sequence found from (a) tactic, the ESG mom this multi-levelled improves functional scoring for query proteins and outperforms previous enzyme functions differ immensely when

improve overall prediction accuracy. One second squiry, the ESG algorithm runs a

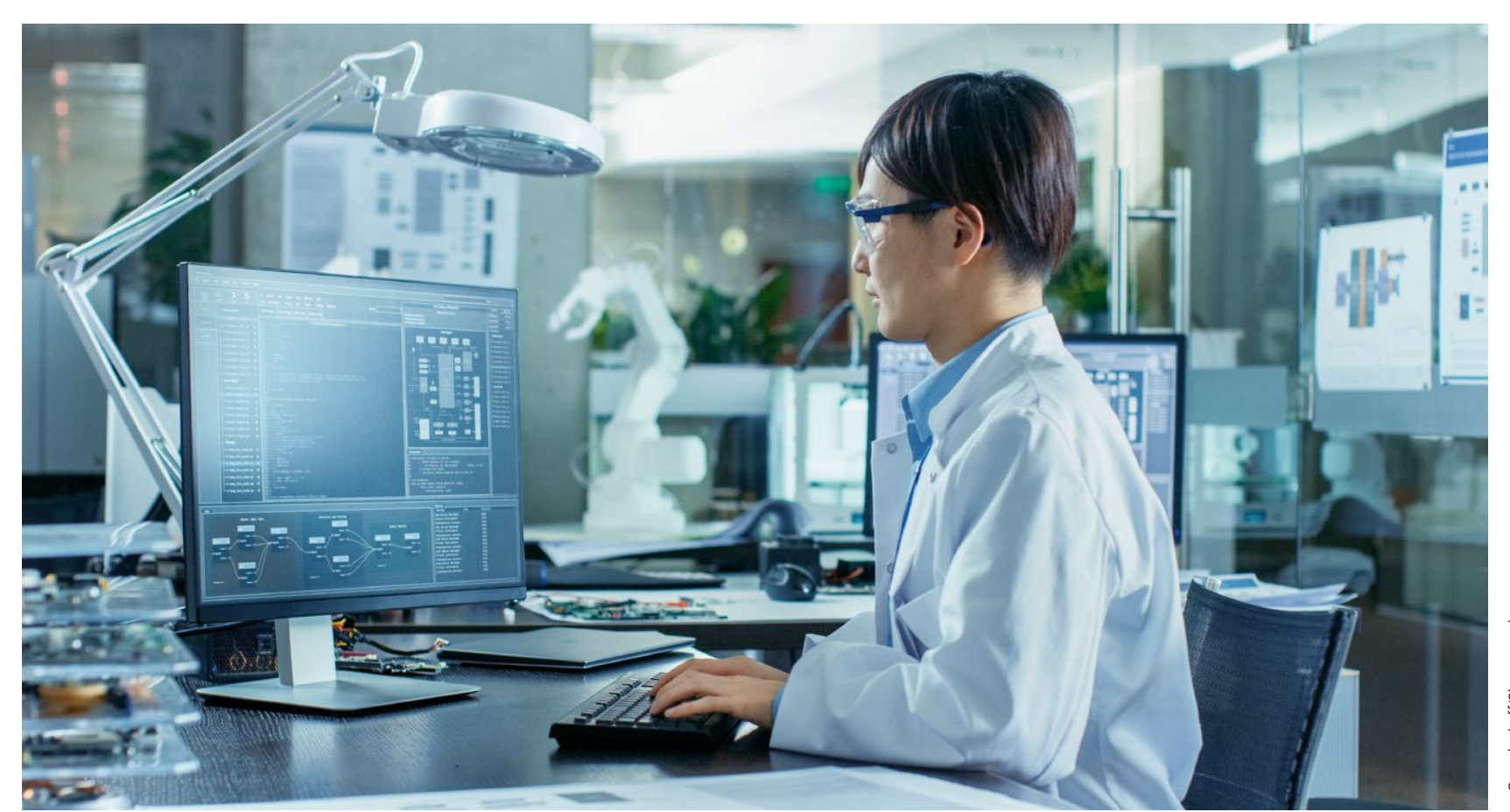

Yet the team did not stop here. In a 2019 paper, they combined phylogenetic sequence-based prediction, called the Phylo-PFP method. They first confirmed that close similarities of protein sequences did not align with the proteins' distances on a phylogenetic tree. By adding these distances into the sequence homology score, the protein query ranks became more reliable, and they could be more accurately linked to their gene source. Unsurprisingly, the study established Phylo-PFP significantly improved existing methods.

\section{PROTEIN GROUP}

\section{UNCTION ANNOTATION}

Protein function annotation is typically run on a one-protein-one-function approach, yet this mindset can grossly oversimplify the protein function universe. In fact, most experiments find dozens of interacting proteins related to a single biological event. To understand the role of an entire protein set, their function should be evernined from the group as a whole, even if the function of each individual protein is unknown. This is no simple task.

Therefore, Dr Kihara's team focused on a new computational approach for annotating the functions of protein groups. In 2019, they proposed an terative Group Function Predction (IGR) logical framework at its core. The iGFP logical framework at its core. The iGFP Moreover, the system automatically
algorithm considers a set of proteins as assumes that some proteins are unknow of the entire group, as well as its individual and uses a range of other comparative members. The iGFP algorithm blends sequence data from multiple sources and builds a complementary network. The method then separates the proteins and compares them based on functional and interaction relationships.

Dr Daisuke Kihara from Purdue University develops function prediction methods with new logical frameworks.

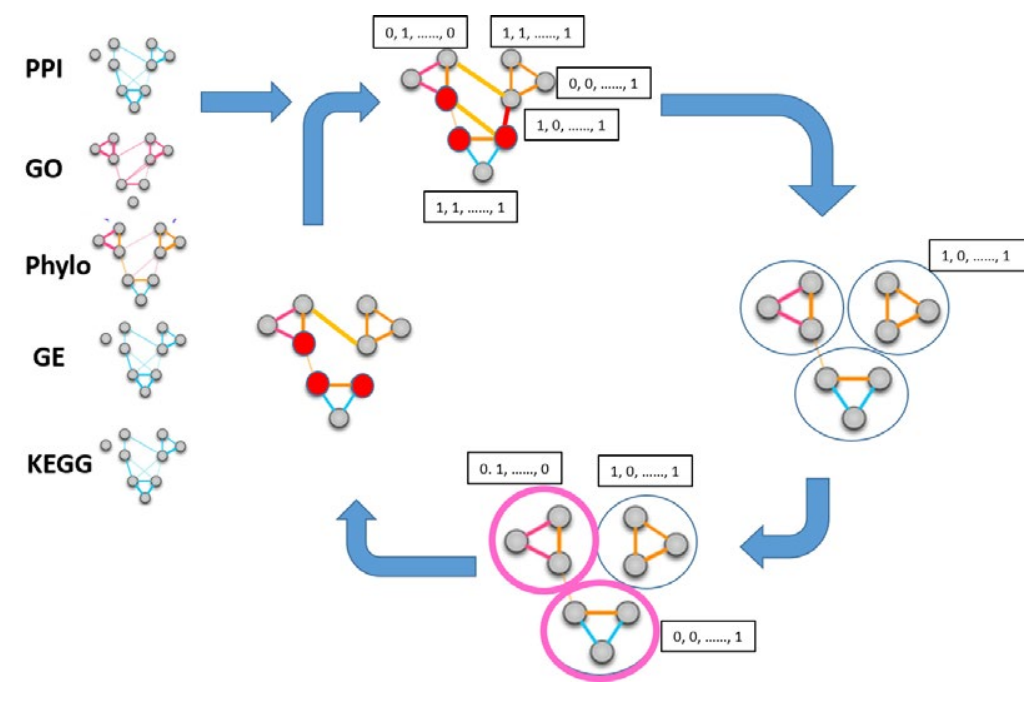

The iGFP algorithm iteratively assigns functions to protein groups and to individual proteins in the groups 


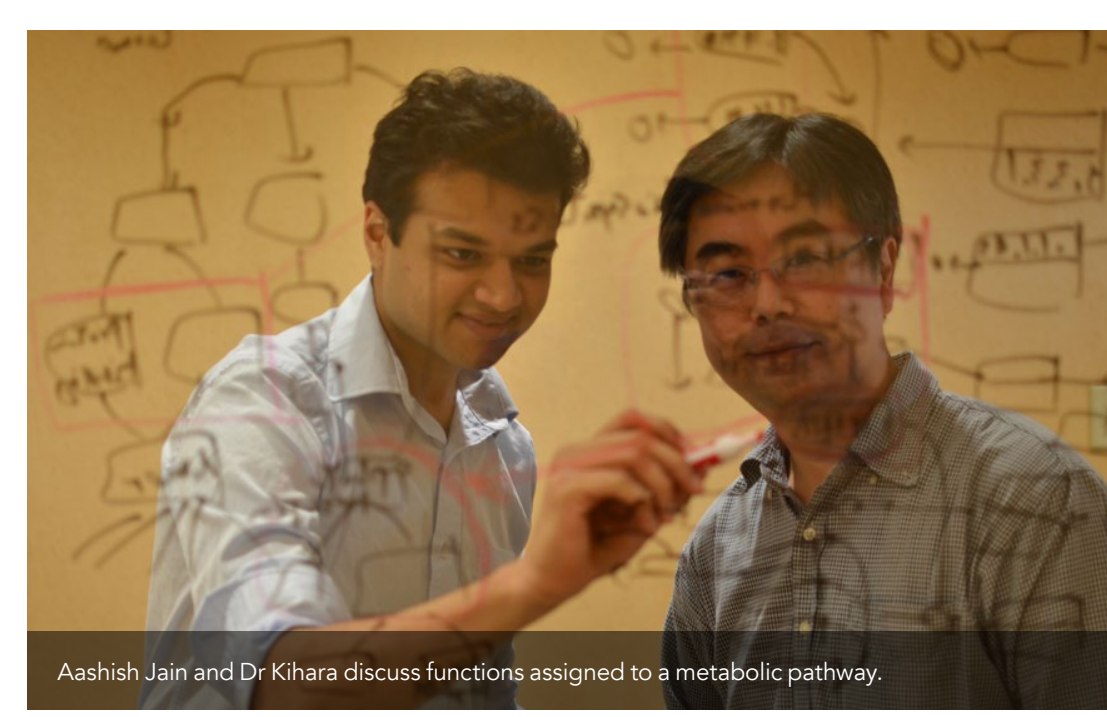

reflection of the real mechanisms at work in, for example, developm or disease-causing pathways.

\section{IDENTIFYING PROTEINS} WITH MULTIPLE FUNCTIONS In addition to analysing protein groups, step away from the one-protein-ontfunction scheme by studying multifunctional proteins. Most bioinformatic tools do not take into account that proteins, enzymes in particular, can be multi-functional. The Kihara lab has thus aimed to predict whether a query protein is a moonlighting protein - one that has multiple autonomous and often unrelated functions. These proteins are difficult to annotate, since their functions are not genome or protein family specific, nor linked to other indicators, such as a shared switching mechanism.

cellular disease states such as cancers, and so identifying them is important. To solve the problem, Dr Kihara's team has developed a new systematic approach to study moonlighting an automated prediction framework which uses several non-sequence-based data to identify moonlighting proteins. They used machine learning classifiers to predict multi-functional proteins, after which they cross validated the results using existing databases. Dr Kihara's team could predict moonlighting proteins that had previous gene sequence data with $98 \%$ accuracy. Even if no sequence data was availab the system showed an impressive

Furthermore, in a 2018 paper the team used deep learning to sniff

The iGFP algorithm considers a set of proteins as input and predicts the function of the entire group, as well as its individual proteins.

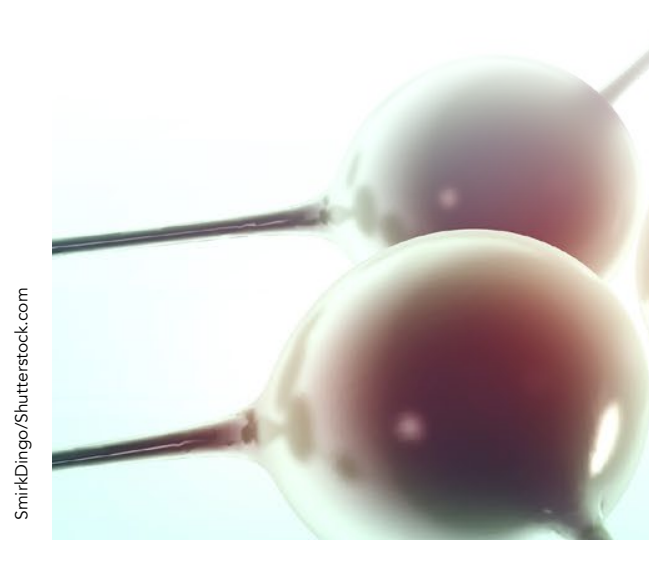

out moonlighting proteins from previously published literature. Their out whether a protein had multiple functions or not based on information from journal publications and functional descriptions from protein databases. Using systematic literature processing tools, the researchers could significantly reduce time to annotate moonlighting proteins and move closer to clarifying the complex interplay of proteins within the cell.

\section{MPROVEMENTS AND}

\section{FUTURE PREDICTIONS}

Computational biology desperately needs new ways to accurately reflect the true nature of biological processes. strides to step away from a tradition strides to step away from a tradiliona and identified functions for entire protein groups. Their algorithms outperform previous sequence-based methods by layering multiple protein characteristics and taking into account evolutionary relationships, which can be better indicators of shared functions than the simple amino acid backbone. Further, the team's machine learning methods can predict whether a protein serves a double role, and whether such proteins have unknowingly been described in previous literature.

Despite these promising developments, bioinformatic prediction tools are there is still a way to go towards fully there is still a way to go towards fully function annotation. Overall. Dr Kihara's team suggests that combining previous methods with emerging ones from omics experiments and evolution distance analysis will further solidify functional prediction accuracies in the future.

\section{0) Behind the Research}

\section{Dr Daisuke Kihara}

E: dkihara@purdue.edu T: +17654962284 W: http://kiharalab.org

\section{Research Objectives}

Dr Kihara's work focuses on developing new techniques for computational protein function prediction.

\section{Detail}

Department of Biological Sciences

Department of Computer Science

249 S. Martin Jischke Dr

West Lafayette, IN 47907, USA

Bio

Dr Kihara is a full professor in the Department of Biologica Sciences and the Department of Computer Science at Purdue University, West Lafayette, Indiana. He received a BS degree from the University of Tokyo, Japan in 1994, and a PhD degree from Kyoto University, Japan in 1999. After studying as a postdoctoral researcher with Prof Jeffrey Skolnick he joined Purdue University in 2003. He was promoted to full professor in 2014. Since 2018, he has held an adjunct professor position at Department of Pediatrics, University of Cincinnati. He has been working in various topics in protein bioinformatics. His current research projects include the developments of algorithm for protein-protein docking, protein tertiary structure prediction, structure modelling from low resolution function prediction and computationat He has published over 150 research papers and book chapters. His research projects have been supported by funding from the National Institutes of $\mathrm{Health}$, the Nation Science Foundation, the Office of the Director of National Intelligence, and industry. He has served on the program committee of various bioinformatics conferences including the Intelligent Systems for Molecular Biology (ISMB) where he is a track chair in 2019. In 2013, he was named a University Faculty Scholar by Purdue University.

Funding

- National Science Foundation (NSF)

- National Institutes of Health (NII)

Collaborators

- Aashish Jain

\section{References}

Chitale, M., Hawkins, T., Park, C., \& Kihara, D. (2009). ESG function prediction. Bioinformatics, 25(14), 1739-1745.

Jain, A., \& Kihara, D. (2019). Phylo-PFP: improved automated protein function prediction using phylogenetic distance

Jain, A., Gali, H., \& Kihara, D. (2018). Identification of Moonlighting Proteins in Genomes Using Text Mining Techniques. Proteomics, 18(21-22), 1800083.

Khan, I. K., \& Kihara, D. (2016). Genome-scale prediction of moonlighting proteins using diverse protein association information. Bioinformatics, 32(15), 2281-2288.

Khan, I. K., Jain, A., Rawi, R., Bensmail, H., \& Kihara, D. (2019). Prediction of protein group function by iterative classification on functional relevance network. Bioinformatics, 8, 1388-1394.

\section{Personal Response}

What kind of role will machine learning play in protein function prediction and understanding biological processes?

II Machine learning has already been playing a big role in protein function prediction, and more widely, subtle signatures that are easily overlooked by humans in input data including protein sequences that are for integrating many different types of data together to make predictions.

PURDUE 